Mannheimer Manuskripte 135/92

January 1992

\title{
The Existence of Solutions \\ of a General Boundary Value Problem \\ FOR THE DIVERGENCE
}

$$
135 / 92
$$

G. SCHWARZ

Lehrstuhl für Mathematik I

Universität Mannheim

Schloß, D - 6800 Mannheim

W. - Germany

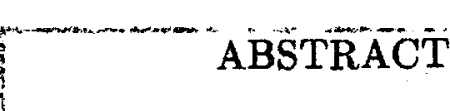

This paper is concerned with the question of necessary and sufficient conditions to find a vector field $V \in \Gamma(T M)$ solving the equation $\operatorname{div} V=\Phi$ under inhomogenous boundary conditions $\left.V\right|_{\partial M}=\left.Z\right|_{\partial M}$ with $Z \in \Gamma(T M)$. An existence and regularity result is given for an arbitrary Riemannian manifold with boundary, $M$. The proof is based on the Hodge theory of differential forms. 


\section{Introduction}

Boundary value problems for differential forms on manifolds $M$ have been investigated for a long time mainly with respect to the question of harmonic solutions, cf. [DuSp,Mo56,Kre]. These results easily apply to solve a problem with the divergence of a vector field and its normal component prescribed in the interior of $M$ and on the boundary $\partial M$, respectively. The solvability of that problem is not spoiled for special mixed problem, one may e.g. prescribe in 3 dimensions also the curl of the vector field, cf. [Duf,Mar]. Few credit, however, has been paid to the general boundary value problem

$$
\begin{array}{ll}
\operatorname{div} V=\Phi & \text { on } M \\
\left.V\right|_{\partial M}=\left.Z\right|_{\partial M} & \text { on } \partial M
\end{array}
$$

The homogeneous case $\left.Z\right|_{\partial M} \equiv 0$ is studied in the literature under certain topological restrictions, e.g. by demanding $M$ to be an open subset of $\mathbb{R}^{3}$ [vWa]. This paper is concerned with the question of existence of solutions of the general problem on an arbitrary Riemannian manifold with boundary. As a central ingredient we apply the harmonic analysis for differential forms, mentioned above, i.e. their Hodge decomposition and results about the harmonic extension of boundary values. Dropping the restriction to harmonic forms gives enough freedom to prove the solvability of (1.1). The analysis is carried out in the $H^{1}$-Sobolev category and regularity results are shown.

Following chapter 7 of [Mor62] we investigate the analytic and geometric structure of the algebra $\Omega(M)$ of differential forms on a bounded Riemannian manifold $M$ in section 2 . Considering the boundary value $\left.\omega\right|_{\partial M}$ of some $\omega \in \Omega^{r}(M)$ we introduce the notion of its normal and tangential components $\mathbf{n} \omega$ and $\mathbf{t} \omega$, respectively. We give a special description for the co-differential operator $\delta$ and rewrite Stoke's theorem in terms of one forms.

In section 3 we study extension problems for differential forms and the Hodge decomposition of functions in that context. Especially we show that the extension problem $\mathbf{t}(\delta \omega)=\left.\zeta\right|_{\partial M}$ with $\left.\omega\right|_{\partial M}=0$ has a solution $\omega \in \Omega^{r}(M)$ for any $\zeta \in \Omega^{r-1}(M)$. We quote Morrey's generalization of the Hodge-Kodeira theorem to manifolds with boundaries, yielding for any $\Phi \in L^{2}(M)$ a decomposition $\Phi=\delta \alpha+C$ such that $\alpha(\mathcal{N})=0$ and $C \in \mathbb{R}$.

By combining the extension result with the Hodge decomposition we prove in section 4 an existence and regularity theorem concerning two important special cases, namely the boundary value problems

$$
\begin{array}{lll}
\delta \beta=\Phi & \text { with } & \beta(\mathcal{N})=\psi \\
\delta \gamma=\Psi & \text { with } & \left.\gamma\right|_{\partial M} \equiv 0
\end{array}
$$

In section 5 a general problem for the co-differential of one forms under inhomogeneous boundary conditions is formulated and its solvability is shown. The divergence and the codifferential operators transform into each other by means of the isomorphism $\sharp: \Gamma(T M) \rightarrow$ $\Omega^{1}(M)$, induced from the metric on $M$, such that $\delta V^{\sharp}=\operatorname{div} V$. As a corollary we then prove the solvability of the boundary value problem (1.1) under the integrability condition 


$$
\int_{M} \Phi \mu_{M}+\int_{\partial M} G_{M}\left(\left.Z\right|_{\partial M}, \mathcal{N}\right) \mu_{\partial}=0
$$

\section{Differential forms on Riemannian manifolds with boundaries}

The study of differential forms on Riemannian manifolds with boundaries goes back to investigations of Duff, Spencer, Conner and Morrey [DuSp,Con,Mo56] in the 50th. While these authors mainly have been interested in solving boundary value problems for harmonic forms we are less restrictive and apply the coresponding results to (linear) problems of a rather different kind. To fix the notion we give the following definitions :

By a $n$-dimensional manifold $M$ with boundary we mean a paracompact topological Hausdorff space, which is locally homeomorphic to an open subset of $\mathbb{R}_{+}^{n}=\left\{\mathbf{x} \in \mathbb{R}^{n} \mid x_{n} \geq 0\right\}$, such that boundary points $p \in \partial M$ are mapped to $\mathbb{R}_{0}^{n}=\left\{\mathbf{x} \in \mathbb{R}^{n} \mid x_{n}=0\right\}$. We call $M$ to be of class $C_{\nu}^{k+1}(k \geq 0$ and $0<\nu \leq 1$, or $k=\infty)$ iff there is an atlas such that of the overlap maps are $C_{\nu}^{\bar{k}+1}$ Hölder smooth with respect to the differentiable structure on $\mathbb{R}_{+}^{n}$, naturally induced from $\mathbb{R}^{n}$ by restriction. In terms of a $C_{\nu}^{k+1}$-atlas we speak about $C_{\nu}^{k+1}$-maps and it is clear how to obtain a $C_{\nu}^{k+1}$-partition of the unite on the bounded manifold $M$.

Vector fields $Y \in \Gamma(T M)$ on $M$ are (at most) of class $C_{\nu}^{k}$ and we can construct a Riemannian metric $G_{M}$ of the same differentiability class. By an unite normal field $\mathcal{N}$ we mean a $C_{\nu}^{k}$-map, which orthogonal to the boundary $\partial M$, i.e. which obeys $G_{M}(\mathcal{N}, Y)(p)=0$ at each point $p \in \partial M$ for all $Y \in \Gamma(T \partial M)$.

For sake of simplicity we restrict ourselves to compact, orientable manifolds with boundary which are at least of class $C_{1}^{1}$. Then the metric $G_{M}$ induces a Riemannian volume form $\mu_{M}$, which can be integrated over $M$ and the normal field $\mathcal{N}$ induces a Riemannian volume element on $\partial M$ given by $\mu_{\partial}:=\mathbf{i}_{\mathcal{N}} \mu_{M}$, where $\mathbf{i}$ is the interior product, cf. [AMR].

Differential forms $\omega \in \Omega^{r}(M)$ are locally, i.e. on $U \subset M$, described by

$$
\varphi^{*}\left(\left.\omega\right|_{U}\right)(p)=\sum_{1 \leq j_{1}<\ldots<j_{r} \leq n} \omega_{\varphi}^{j_{1}, \ldots, j_{r}}\left(y_{1}, \ldots, y_{n}\right) d x^{j_{1}} \wedge \ldots \wedge d x^{j_{r}}
$$

where $\varphi(p)=\left(y_{1}, \ldots, y_{n}\right)$ and $\left\{d x^{0}, \ldots d x^{n}\right\}$ is the standard dual base of $\mathbb{R}^{n+1} . \omega$ is called a differential form of class $C_{\nu}^{k}$, if the coefficient functions $\omega_{\varphi}^{j_{1}, \ldots, j_{r}}$ are of class $C_{\nu}^{k}$ on $\varphi(U) \subset \mathbb{R}_{+}^{n}$ for any chart $(U, \varphi)$. For the boundary value of $\omega \in \Omega^{r}(M)$, i.e. its restriction $\omega \mid \partial M$, we have in a chart $(U, \varphi)$

$$
\varphi^{*}\left(\left.\omega\right|_{\partial M \cap U}\right)(p)=\sum_{1 \leq j_{1}<\ldots<j_{r} \leq n} \omega_{\varphi}^{j_{1}, \ldots, j_{r}}\left(y_{1}, \ldots, y_{n-1}, 0\right) d x^{j_{1}} \wedge \ldots \wedge d x^{j_{r}}
$$

with $\varphi(p)=\left(y_{1}, \ldots, y_{n-1}, 0\right)$ for $p \in \partial M .\left.\omega\right|_{\partial M}$ is called of class $C_{\nu}^{k}$ along $\partial M$, iff for any chart the restriction $\left.\left(\omega_{\varphi}^{j_{1}, \ldots, j_{r}}\right)\right|_{\mathbb{R}_{0}^{n}}$ of the coefficient function is of that class with respect to the differentiable structure on $\mathbb{R}^{n-1}$.

The boundary value $\left.\omega\right|_{\partial M}$ naturally splits into a tangential and a normal component 


$$
\left.\omega\right|_{\partial M}=\mathbf{t} \omega+\mathbf{n} \omega
$$

such that $\operatorname{t} \omega\left(\mathcal{N}, Y_{1}, \ldots Y_{r-1}\right)=0$ for all fields $Y_{k} \in \Gamma\left(\left.T M\right|_{\partial M}\right)$. For the normal component we have in a local representation

$$
\varphi^{*}\left(\left.\mathbf{n} \omega\right|_{U}\right)(p)=\sum_{1 \leq j_{1}<\ldots<j_{r-1} \leq n-1} \omega_{\varphi}^{j_{1}, \ldots, j_{r-1}, n}\left(y_{1}, \ldots, y_{n-1}, 0\right) d x^{j_{1}} \wedge \ldots \wedge d x^{j_{r-1}} \wedge d x^{n}
$$

The exterior algebra $\Omega(M)$ is constructed in the same way as for manifolds without boundary, i.e. the $\wedge$-product and the exterior derivative $d: \Omega^{r}(M) \rightarrow \Omega^{r+1}(M)$ compute as usual. The Hodge operator $\star: \Omega^{r}(M) \rightarrow \Omega^{n-r}(M)$ becomes, written in coordinates

$$
(\star \omega)_{\varphi}^{i_{1}, \ldots, i_{n-r}}=\omega_{\varphi}^{j_{1}, \ldots, j_{r}}
$$

where the (ordered) tuple $\left[i_{1}, \ldots, i_{n-r}\right]$ is the complement of $\left[j_{1}, \ldots, j_{r}\right]$ in the set $[1, \ldots, n]$ For the boundary values the identity $\left.(\star \omega)\right|_{\partial M}=\star\left(\left.\omega\right|_{\partial M}\right)$ is obvious and we get

$$
\left.(\star \omega)\right|_{\partial M}=\mathbf{n}(\star \omega)+\star(\mathbf{n} \omega)
$$

From (2.4) and (2.5) it is easily seen that

$$
\left(\left.\omega\right|_{\partial M}\right)_{\varphi}^{j_{1}, \ldots, j_{r}}= \begin{cases}(\mathbf{n} \star \omega)_{\varphi}^{i_{1}, \ldots, i_{n-r}} & \text { where } i_{n-r}=n \\ (\star \mathbf{n} \omega)_{\varphi}^{i_{1}, \ldots, i_{n-r}} & \text { where } j_{r}=n\end{cases}
$$

Since $\left[i_{1}, \ldots, i_{n-r}\right]$ and $\left[j_{1}, \ldots, j_{r}\right]$ are complementary to each other in both cases we have $i_{n-r} \neq j_{r}$ if and only if $j_{r}=n$, what proves (2.6).

By means of the Hodge operator the co-differential $\delta: \Omega^{r+1}(M) \rightarrow \Omega^{r}(M)$ is given as $\delta:=(-1)^{n r+1} \star d \star$. Following [Mat] it computes in terms of a local $G_{M}$-orthonormal frame $\left\{E_{1}, \ldots, E_{n}\right\}$ on $T M$ and the coresponding Levi-Civita connection $\nabla$ as

$$
(\delta \omega)\left(X_{1}, \ldots X_{r}\right)=-\sum_{k=1}^{n}\left(\nabla_{E_{k}} \omega\right)\left(E_{k}, X_{1}, \ldots X_{r}\right) \quad \text { with } \quad X_{1}, \ldots X_{r} \in \Gamma(T M)
$$

Finally we introduce a Riemannian structure on $\Omega^{r}(M)$ given by

$$
\omega \bullet \eta:=\int_{M} \omega \wedge \star \eta
$$

Since $M$ is compact by assumption the integral converges. Using the coresponding norm $\|\cdot\|$ we equip $\Omega^{r}(M)$ with a Sobolev structure : The Sobolev spaces $H^{0} \Omega^{r}(M)$ and $H^{1} \Omega^{r}(M)$ are defined as the completion of $\Omega^{r}(M)$ with respect to the norms $\|\omega\|$ and $\sqrt{\|\omega\|^{2}+\|d \omega\|^{2}}$, respectively. We furthermore call the boundary value $\left.\omega\right|_{\partial M}$ to be of class $H^{1}$ along $\partial M$, iff the restrictions $\left.\left(\omega_{\varphi}^{j_{1}, \ldots, j_{r}}\right)\right|_{\mathbb{R}_{0}^{n}}$ are of class $H^{1}\left(\mathbb{R}^{n-1}\right)$ for any chart, intersecting $\partial M$. 
In the $H^{1}$-category we then have Stoke's theorem [AMR], which yields for the special case of one forms :

$$
\int_{M} \delta \omega \mu_{M}=-\int_{\partial M} \omega(\mathcal{N}) \mu_{\partial}
$$

\section{Extension problems for differential forms and Hodge theory for functions}

By an extension problem we mean the question of existence of a $r$-form $\omega \in \Omega^{r}(M)$ - of a certain differentiability class on $M$ - with some components of its boundary value or of the boundary value of its derivative prescribed. Denoting by $D^{m}$ the unite disk in $\mathbb{R}^{m}$, the analytic foundation for the problems, we have in mind, is laid by the following lemma:

\section{Lemma 1}

For any $f \in H^{1}\left(\mathbb{R}^{n-1}\right)$ with $\operatorname{supp}(f) \subset D^{n-1}$ there exists a map $u: \mathbb{R}^{n} \rightarrow \mathbb{R}$ with $u \in H^{1}\left(\mathbb{R}^{n}\right)$ and $\operatorname{supp}(u) \subset D^{n}$ such that

$$
\begin{aligned}
u\left(y_{1}, \ldots, y_{n-1}, 0\right) & =0 \\
\partial_{y_{n}} u\left(y_{1}, \ldots, y_{n-1}, 0\right) & =f\left(y_{1}, \ldots, y_{n-1}\right)
\end{aligned}
$$

All derivatives $\partial_{y_{j}} u$ can be chosen of class $H^{1}\left(\mathbb{R}^{n}\right)$. If $f \in C_{\nu}^{k}\left(\mathbb{R}^{n-1}\right)$ the map $u$ can be chosen of class $C_{\nu}^{k+1}\left(\mathbb{R}^{n}\right)$.

For a proof we refer to section 6 of [Mo56]. Inspired by results from the same work of Morrey one shows :

\section{Lemma 2}

Let $M$ be a Riemannian manifold with boundary of class $C_{1}^{1}$ and let $\eta \in \Omega^{r}(M)$ be a $r$-form with boundary value $\left.\eta\right|_{\partial M}$ of class $H^{1}$ on $\partial M$.

a) There exists a $H^{1}$-extension of $\left.\eta\right|_{\partial M}$, i.e. a $r$-form $\chi \in H^{1} \Omega^{r}(M)$, such that

$$
\left.\chi\right|_{\partial M}=\left.\eta\right|_{\partial M}
$$

b) There exists a $(r-1)$-form $\omega \in H^{1} \Omega^{r-1}(M)$ with $d \omega \in H^{1} \Omega^{r}(M)$ such that

$$
\left.\omega\right|_{\partial M}=0 \quad \text { and } \quad \mathbf{n}(d \omega)=\mathbf{n} \eta
$$

If $\mathrm{M}$ is of class $C_{\nu}^{k+1}$ with $k \geq 1$ and $\left.\eta\right|_{\partial M}$ is of class $C_{\nu}^{k}$ on $\partial M$, then $\chi$ can be chosen of class $C_{\nu}^{k}$ on $M$ and $\omega$ can be chosen such that $\omega$ and $d \omega$ are of class $C_{\nu}^{k}$ on $M$. 
Proof :

a) Let $\left\{\left(U_{\alpha}, \varphi_{\alpha}\right)\right\}_{\alpha \in A}$ be an atlas on $M$ and $\left\{\rho_{\alpha}\right\}_{\alpha \in A}$ a subordinated $C_{\nu}^{k+1}$ partition of unite. For each $\alpha \in A$ we define a $r$-form $\chi_{\alpha} \in H^{1} \Omega^{r}(M)$ by

$$
\chi_{\alpha}:= \begin{cases}0 & \text { if } \operatorname{supp}\left(\rho_{\alpha}\right) \cap \partial M=\emptyset \\ \tilde{\chi}_{\alpha} & \text { else }\end{cases}
$$

where $\left.\tilde{\chi}_{\alpha}\right|_{M \backslash U_{\alpha}}:=0$ and $\left.\tilde{\chi}_{\alpha}\right|_{U_{\alpha}}$ is locally determined from $\eta$ by

$$
\left(\tilde{\chi}_{\alpha}\right)_{\varphi_{\alpha}}^{j_{1}, \ldots, j_{r}}\left(y_{1}, \ldots, y_{n}\right)=\eta_{\varphi_{\alpha}}^{j_{1}, \ldots, j_{r}}\left(y_{1}, \ldots, y_{n-1}, 0\right) \cdot\left(\rho_{\alpha} \circ \varphi_{\alpha}^{-1}\right)\left(y_{1}, \ldots, y_{n}\right)
$$

Setting $\chi:=\sum_{\alpha \in A} \chi_{\alpha}$, we get a solution of (3.2) of the desired differentiability class.

b) Let $\left\{\left(U_{\alpha}, \varphi_{\alpha}\right)\right\}_{\alpha \in A}$ be an atlas on $M$ such that $\varphi_{\alpha}\left(U_{\alpha}\right) \supset D^{n}$. Given a subordinated $C_{\nu}^{k+1}$-partition of unite $\left\{\rho_{\alpha}\right\}_{\alpha \in A}$, which obeys $\varphi_{\alpha}\left(\operatorname{supp}\left(\rho_{\alpha}\right) \cap \partial M\right) \subset D^{n-1}$. We define for each $\alpha \in A$ a $r$-form $\omega_{\alpha} \in H^{1} \Omega^{r}(M)$ by

$$
\omega_{\alpha}:= \begin{cases}0 & \text { if } \operatorname{supp}\left(\rho_{\alpha}\right) \cap \partial M=\emptyset \\ \widetilde{\omega}_{\alpha} & \text { else }\end{cases}
$$

where $\left.\widetilde{\omega}_{\alpha}\right|_{M \backslash U_{\alpha}}:=0$ and $\left.\widetilde{\omega}_{\alpha}\right|_{U_{\alpha}}$ is locally determined from $\eta$ as follows: We set $\eta_{\alpha}:=\eta \cdot \rho_{\alpha}$ and know from lemma 1 that for all $1 \leq j_{1}<\ldots<j_{r-1} \leq n$ the problem

$$
\begin{aligned}
h_{\alpha}^{j_{1}, \ldots, j_{r-1}}\left(y_{1}, \ldots, y_{n-1}, 0\right) & =0 \\
\partial_{y_{n}} h_{\alpha}^{j_{1}, \ldots, j_{r-1}}\left(y_{1}, \ldots, y_{n-1}, 0\right) & =\left(\eta_{\alpha}\right)_{\varphi_{\alpha}}^{j_{1}, \ldots, j_{r-1}, n}\left(y_{1}, \ldots, y_{n-1}, 0\right)
\end{aligned}
$$

has a solution $h_{\alpha}^{j_{1}, \ldots, j_{r-1}} \in H^{1}\left(\mathbb{R}^{n}\right)$ with support in the interior of $D^{n}$. Furthermore these solutions can be chosen of class $C_{\nu}^{k+1}$ on $M$; if $\left.\eta\right|_{\partial M}$ is of class $C_{\nu}^{k}$. By construction $h_{\alpha}^{j_{1}, \ldots, j_{r-1}}$ can be considered (in the sense of (2.1)) as the coefficient function of a $(r-1)$-form, since it transforms properly under change of charts. Hence we define $\left(\left.\widetilde{\omega}_{\alpha}\right|_{U_{\alpha}}\right)_{\varphi_{\alpha}}^{j_{1}, \ldots, j_{r-1}}:=h_{\alpha}^{j_{1}, \ldots, j_{r-1}}$ what solves (for each $\alpha \in A$ ) the problem

$$
\left.\widetilde{\omega}_{\alpha}\right|_{\partial M}=0 \quad \text { and } \quad \mathbf{n}\left(\left.d \widetilde{\omega}_{\alpha}\right|_{\partial M \cap U_{\alpha}}\right)=\mathbf{n}\left(\left.\eta_{\alpha}\right|_{\partial M \cap U_{\alpha}}\right)
$$

Setting $\omega:=\sum_{\alpha \in A} \omega_{\alpha}$, we get a solution of (3.3) of the desired differentiability class.

For our further investigations we need the (Hodge) dual of the result in part b :

\section{Corollary 3}

Let $M$ be as in lemma 2. Given $\zeta \in \Omega^{r-1}(M)$ with $\zeta l_{\partial M}$ of class $H^{1}$ on $\partial M$, there exists some $\chi \in H^{1} \Omega^{r}(M)$ with $\delta \chi \in H^{1} \Omega^{r-1}(M)$ obeying the boundary conditions

$$
\left.\chi\right|_{\partial M}=0 \quad \text { and } \quad \mathbf{t}(\delta \chi)=\mathbf{t} \zeta
$$

If $\left.\zeta\right|_{\partial M}$ is of class $C_{\nu}^{k}$ on $M$ then $\chi$ and $\delta \chi$ can be chosen of class $C_{\nu}^{k}$ on $M$, too. 
Proof :

For $\eta=\star \zeta$ the problem (3.3) transforms by means of (2.6) into

$$
\left.\omega\right|_{\partial M}=0 \quad \text { and } \quad \mathbf{n}(d \omega)=\star(\mathbf{t} \zeta)
$$

Taking the Hodge dual of this and using (2.6) once more we get

$$
\left.\star \omega\right|_{\partial M}=0 \quad \text { and }\left.\quad \star(d \omega)\right|_{\partial M}-\mathbf{n}(\star d \omega)=(-1)^{n r+1} \mathbf{t} \zeta
$$

Hence $\chi:=\star \omega$ solves the problem (3.9), iff $\omega$ is a solution of (3.3). Since $\star$ is an operator of class $C_{\nu}^{k}$, this solution is of the desired differentiability class, too.

The central result to face boundary value problems for differential forms is Morrey's generalization of the Hodge-Kodeira decomposition theorem for manifolds with boundaries, cf. chapter 7.7 of [Mo62] :

\section{Theorem 4}

Let $M$ be a compact Riemannian $C_{\nu}^{k+1}$-manifold with boundary where $(k, \nu)=(0,1)$ or $k \geq 1$ and $0<\nu<1$ and denote by $\mathcal{E}^{r}, \mathcal{D}^{r}$ and $\mathcal{H}^{r}$ the following subspaces of $H^{0} \Omega^{r}(M)$ :

$$
\begin{aligned}
\mathcal{E}^{r} & :=\left\{\delta \alpha \mid \alpha \in H^{1} \Omega^{r+1}(M) \text { with } \mathbf{n} \alpha=0\right\} \\
\mathcal{D}^{r} & :=\left\{d \beta \mid \beta \in H^{1} \Omega^{r-1}(M) \text { with } \mathbf{t} \beta=0\right\} \\
\mathcal{H}^{r} & :=\left\{\kappa \in H^{1} \Omega^{r}(M) \mid \text { with } d \kappa=\delta \kappa=0\right\}
\end{aligned}
$$

There is an orthonormal decomposition

$$
H^{0} \Omega^{r}(M)=\mathcal{E}^{r} \oplus \mathcal{D}^{r} \oplus \mathcal{H}^{r}
$$

with respect to the product (2.9) on $H^{0} \Omega^{r}(M)$. Each $\omega \in H^{0} \Omega^{r}(M)$ uniquely writes as

$$
\omega=\delta \alpha+d \beta+\kappa
$$

such that $\delta \alpha \in \mathcal{E}^{r}, d \beta \in \mathcal{D}^{r}$ and $\kappa \in \mathcal{H}^{r}$. If $\omega \in \Omega^{r}(M)$ is of class $C_{\nu}^{k-1}$ (for $k \geq 1$ ), $\alpha$ and $\beta$ can be chosen of class $C_{\nu}^{k}$ and $\kappa$ of class $C_{\nu}^{k-1}$.

For our purpose it is important to consider the special case of zero forms, i.e. the case of usual (Lebegue-) functions $\Phi \in L^{2}(M)$, where the Morrey-Kodeira decomposition yields

$$
\Phi=\delta \alpha+C \quad \text { with } \alpha \in H^{1} \Omega^{1}(M) \text { such that } \alpha(\mathcal{N})=0 \text { and } C \in \mathbb{R}
$$

This is obvious since $\mathcal{D}^{0}=\{0\}$ and $\mathcal{H}^{0}=\mathbb{R}$ for any manifold $M$. The regularity results of theorem 4 hold accordingly. 


\section{Special boundary value problems for one forms}

Now we combine the (classical) results, presented in the preceding section, to solve boundary value problems for one forms. We are interested in first order problems, involving the co-differential operator $\delta$ and consider at first Neumann and homogeneous boundary conditions :

\section{Lemma 5}

Let $M$ be a compact Riemannian $C_{\nu}^{k+1}$-manifold with boundary, where $(k, \nu)=(0,1)$ or $k \geq 1$ and $0<\nu<1$.

a) Given a pair of functions $\Phi \in L^{2}(M)$ and $\psi \in H^{1}(\partial M)$, which obey the integrability condition

$$
\int_{M} \Phi \mu_{M}+\int_{\partial M} \psi \mu_{\partial}=0
$$

there exists a one form $\beta \in H^{1} \Omega^{1}(M)$ which solves the boundary value problem

$$
\begin{aligned}
\delta \beta=\Phi & \text { on } M \\
\beta(\mathcal{N})=\psi & \text { on } \partial M
\end{aligned}
$$

If $\Phi \in C_{\nu}^{k-1}(M)$ and $\psi \in C_{\nu}^{k}(\partial M)$ (for $k \geq 1$ ) one can chose $\beta$ of class $C_{\nu}^{k}$ on $M$.

b) Given a function $\Psi \in H^{1}(M)$ such that $\left.\Psi\right|_{\partial M} \in H^{1}(\partial M)$, which obeys the integrability condition

$$
\int_{M} \Psi \dot{\mu}_{M}=0
$$

there exists a one form $\gamma \in H^{1} \Omega^{1}(M)$ which solves the boundary value problem

$$
\begin{aligned}
\delta \gamma & =\Psi \quad \text { on } M \\
\left.\gamma\right|_{\partial M} & \equiv 0 \quad \text { on } \quad \partial M
\end{aligned}
$$

If $\Psi \in C_{\nu}^{k-1}(M)$ (for $k \geq 1$ ) one can chose $\gamma$ of class $C_{\nu}^{k}$ on $M$.

Proof :

a) Applying the Morrey-Kodeira decomposition (3.14) to $\Phi \in L^{2}(M)$, determines some $\alpha_{\Phi} \in H^{1} \Omega^{1}(M)$ such that

$$
\Phi=\delta \alpha_{\Phi}+C_{\Phi} \quad \text { with } \quad \alpha_{\Phi}(\mathcal{N})=0
$$

On the other hand lemma 2 and the collar theorem, cf. [Hir], guaranty for any $\psi \in H^{1}(\partial M)$ the existence of some $\eta_{\psi} \in H^{1} \Omega^{1}(M)$ such that $\left.\eta_{\psi}\right|_{\partial M}(\mathcal{N})=\psi$ on $\partial M$. Applying (3.14) to the function $\delta \eta_{\psi} \in L^{2}(M)$ then determines some $\alpha_{\psi} \in H^{1}(M)$, such that

$$
\delta \eta_{\psi}=\delta \alpha_{\psi}+C_{\psi} \quad \text { with } \quad \alpha_{\psi}(\mathcal{N})=0
$$


From the regularity results of the Morrey-Kodeira decomposition and lemma 2 we get $\alpha_{\Phi}$ as well as $\eta_{\psi}$ and $\alpha_{\psi}$ being of class $C_{\nu}^{k}$ if $\Phi \in C_{\nu}^{k-1}(M)$ and $\psi \in C_{\nu}^{k}(\partial M)$.

Now Stoke's theorem (2.10) and the integrability condition (4.1) yields

$$
\left(C_{\Phi}-C_{\psi}\right) \cdot \int_{M} \mu_{M}=\int_{M}\left(\Phi-\delta \eta_{\psi}-\delta \alpha_{\Phi}+\delta \alpha_{\psi}\right) \mu_{M}=0
$$

Then $\beta:=\alpha_{\Phi}+\eta_{\psi}-\alpha_{\psi}$ is a solution of (4.2) of the desired regularity class.

b) We start as above and apply (3.14) to the function $\Psi \in H^{1}(M)$. The integrability condition (4.3) implies that the constant $C_{\Psi}$ has to vanish, so we get

$$
\Psi=\delta \alpha_{\Psi} \quad \text { with } \quad \alpha_{\Psi}(\mathcal{N})=0
$$

By assumption $\left.\Psi\right|_{\partial M} \in H^{1}(\partial M)$, hence we have also $\alpha_{\Psi}$ (at least) of class $H^{1}$ along $\partial M$. Then corollary 3 determines a two form $\zeta_{\Psi} \in H^{1} \Omega^{2}(M)$ solving the problem

$$
\left.\zeta_{\Psi}\right|_{\partial M} \equiv 0 \quad \text { and }\left.\quad\left(\delta \zeta_{\Psi}\right)\right|_{\partial M}(X)=\left.\alpha_{\Psi}\right|_{\partial M}(X) \quad \forall X \in \Gamma T \partial M
$$

such that $\delta \zeta_{\Psi} \in H^{1} \Omega^{1}(M)$. For $\Psi \in C_{\nu}^{k-1}(M)$ we furthermore have $\alpha_{\Psi}$ of class $C_{\nu}^{k}$ and hence $\zeta_{\Psi}$ and $\delta \zeta_{\Psi}$ can be chosen of class $C_{\nu}^{k}$ on $M$. Using the fundamental property of the co-differential to be nilpotent, saying that $\delta(\delta \omega)=0$ for all $\omega \in \Omega^{r}(M)$, we see that

$$
\begin{array}{ll}
\delta\left(\alpha \Psi-\delta \zeta_{\Psi}\right)=\Psi & \text { on } M \\
\mathbf{t}\left(\alpha_{\Psi}-\delta \zeta_{\Psi}\right)=0 & \text { on } \partial M
\end{array}
$$

To investigate the normal components we have $\mathbf{n} \alpha_{\Psi} \equiv 0$ by construction. For $\mathbf{n}\left(\delta \zeta_{\Psi}\right)$ we use the collar theorem [Hir], which guaranties near any $p \in \partial M$ the existence of a local orthonormal frame $\left\{\widetilde{\mathcal{N}}, \widetilde{E}_{2}, \ldots, \widetilde{E}_{n}\right\}$ such that $\left.\widetilde{\mathcal{N}}\right|_{\partial M}=\mathcal{N}$ and $\left.\widetilde{E}_{i}\right|_{\partial M}$ is tangential to $\partial M$. Then (2.8) yields

$$
\begin{aligned}
\left.\left(\delta \zeta_{\Psi}\right)\right|_{\partial M}(\mathcal{N}) & =-\left.\left(\nabla_{\widetilde{\mathcal{N}}} \zeta_{\Psi}\right)\right|_{\partial M}(\tilde{\mathcal{N}}, \mathcal{N})-\left.\sum_{k=2}^{n}\left(\nabla_{\widetilde{E}_{k}} \zeta_{\Psi}\right)\right|_{\partial M}\left(\widetilde{E}_{k}, \mathcal{N}\right) \\
& =-\left.\sum_{k=2}^{m} \nabla_{\widetilde{E}_{k}}\left(\zeta_{\Psi}\left(\widetilde{E}_{k}, \mathcal{N}\right)\right)\right|_{\partial M}
\end{aligned}
$$

since $\left.\left(\zeta_{\Psi}\right)\right|_{\partial M} \equiv 0$ by construction. Also due to that fact $\left.\left(\zeta_{\Psi}\right)\right|_{\partial M}$ is covariantly constant in the direction of each $\left.\widetilde{E}_{k}\right|_{\partial M}$, which are vector fields along the boundary $\partial M$. This proves that $\left.\left(\delta \zeta_{\Psi}\right)\right|_{\partial M}(\mathcal{N})=0$. So the boundary value of $\left(\alpha_{\Psi}-\delta \zeta_{\Psi}\right)$ vanishes identical. Hence $\gamma:=\alpha_{\Psi}-\delta \zeta_{\Psi}$ yields a solution of (4.4) of the desired regularity.

Both assertions of that lemma are not original. Part a) may also be derived by transforming the boundary value problem (4.2) into a standard elliptic problem : Demanding $\beta=d \mathcal{H}$ for some function $\mathcal{H}$, we are left with the Neumann problem $\triangle \mathcal{H}=\Phi$ and $d \mathcal{H}(\mathcal{N})=\psi$, which has a unique solution, $\mathrm{cf}$. [Hör]. By using more a subtile extension results [Pal] as we have from lemma 2, also the existence of a solution can be guaranteed if $\psi$ is only of Sobolev class $H^{1 / 2}$ on $\partial M$. 
For part b) there is no elliptic reformulation, i.e. the problem cannot be identified with some standard Dirichlet problem. In the literature [Bog,vWa] there has been considered the existence question for the divergence of vector fields under homogeneous boundary conditions, what is an equivalent to our study on the co-differential of one forms, see section 5 below. We note, however, that the authors were more restrictive in choosing $M$ - considering either the sum of starlike connected domains in $\mathbb{R}^{n}[\mathrm{Bog}]$ or open subsets of $\mathbb{R}^{3}$ [vWa] - but thereby also could give an explicit kernel to solve the problem and describe the precise range of the operator $\delta$. Another approach is due to [Kre] who can find harmonic solutions for the boundary value problem (4.4), but requires stronger integrability conditions. In turn our result - based on a variational method [Mor62] - allows to solve this problem for a general Riemannian manifold but just gives necessary and sufficient conditions for the existence of solutions.

\section{The problem $\operatorname{div} V=\Phi$ under general boundary conditions}

With the same techniques, we used to prove lemma 5, we now can study the solvability of a more general problem :

\section{Theorem 6}

Let $M$ be a compact Riemannian $C_{\nu}^{k+1}$-manifold with boundary, where $(k, \nu)=(0,1)$ or $k \geq 1$ and $0<\nu<1$.

Given a function $\Phi \in L^{2}(M)$ and one form $\Xi \in \Omega^{1}(M)$ with boundary value $\left.\Xi\right|_{\partial M}$ of class $H^{1}$ on $\partial M$, which obey the integrability condition

$$
\int_{M} \Phi \mu_{M}+\int_{\partial M} \Xi(\mathcal{N}) \mu_{\partial}=0
$$

there exists a one form $\alpha \in H^{1} \Omega^{1}(M)$ solving the boundary value problem

$$
\begin{array}{rlrl}
\delta \alpha & =\Phi & & \text { on } M \\
\left.\alpha\right|_{\partial M} & =\left.\Xi\right|_{\partial M} & \text { on } \partial M
\end{array}
$$

If $\Phi \in C_{\nu}^{k-1}(M)$ and $\left.\Xi\right|_{\partial M}$ is of class $C_{\nu}^{k}$ (for $k \geq 1$ ), $\alpha$ can be chosen of class $C_{\nu}^{k}$ on $M$.

Proof :

Since the normal field $\mathcal{N}$ is (at least) of differentiability class $C_{1}^{0}$ on $\partial M$ the function $\psi_{\Xi}:=\Xi(\mathcal{N})$ is of Sobolev class $H^{1} \Omega^{0}(\partial M)$. Under the integrability condition (5.1) part a) of lemma 5 guaranties the existence of a one form $\beta \in H^{1} \Omega^{1}(M)$, such that

$$
\delta \beta=\Phi \quad \text { and }\left.\quad \beta\right|_{\partial M}(\mathcal{N})=\psi \Xi
$$

On the other hand the extension problem

$$
\left.\xi\right|_{\partial M} \equiv 0 \quad \text { and }\left.\quad \mathbf{t}(\delta \xi)\right|_{\partial M}=\mathbf{t}(\Xi-\beta)
$$


has a solution $\xi \in H^{1} \Omega^{2}(M)$ with $\delta \xi \in H^{1} \Omega^{1}(M)$ by using corollary 3 . Furthermore one shows - literally as in the prove of lemma 5 (part b) - that this two-form also obeys $\left.(\delta \xi)\right|_{\partial M}(\mathcal{N})=0$.

Hence we obtain $\alpha:=\beta+\delta \xi$ as a solution of the problem

$$
\delta \alpha=\Phi, \quad \mathbf{n} \alpha=\mathbf{n} \Xi \text { and } \mathbf{t}\left(\left.\alpha\right|_{\partial M}\right)=\mathbf{t}(\Xi)
$$

Finally we conclude from the regularity results of lemma 2 and corollary 3 that $\beta$ and $\delta \xi$ are of class $C_{\nu}^{k}$ if $\Phi \in C_{\nu}^{k-1}(M)$ and $\left.\Xi\right|_{\partial M}$ is of class $C_{\nu}^{k}$.

The key observation for the proof - as for the proves of lemma 5 - was that the codifferential is a nilpotent operator, i.e. $\delta^{2}=0$. This fact made it possible to modify the one form determined from the Hodge decomposition of $\Phi$, which solves a homogeneous boundary value problem, by the co-differential of any two form. Knowing this and having the appropriate extension results at hand we could adjust the solution to the desired boundary value.

Boundary value problems originated from physics, however, are - in their classical formulation - given in terms of vector fields instead of one forms. To make our results applicable we observe that the Riemannian structure $G_{M}$ on a $C_{\nu}^{k+1}$-manifold $M$ induces a $C_{\nu}^{k}$-isomorphism

$$
\begin{aligned}
\sharp: \Gamma(T M) & \longrightarrow \Omega^{1}(M) \\
W^{\sharp}(Y) & :=G_{M}(Y, W) \quad \forall Y \in \Gamma(T M)
\end{aligned}
$$

Under this map the co-differential operator (2.8) transforms into the divergence, i.e.

$$
\delta W^{\sharp}=\operatorname{div} W \quad \forall W \in \Gamma(T M)
$$

and our central result (theorem 6) immediately transforms into

\section{Corollary 7}

Let $M$ be as in theorem 6. Given a function $\Phi \in L^{2}(M)$ and a vector field $Z \in \Gamma(T M)$ with $\left.Z\right|_{\partial M}$ of class $H^{1}$ on $\partial M$, the boundary value problem

$$
\begin{array}{llrl}
\operatorname{div} V & =\Phi & & \text { on } M \\
\left.V\right|_{\partial M}=\left.Z\right|_{\partial M} & \text { on } \partial M
\end{array}
$$

has a solution $V \in \Gamma(T M)$, if and only if the integrability condition

$$
\int_{M} \Phi \mu_{M}+\int_{\partial M} G_{M}\left(\left.Z\right|_{\partial M}, \mathcal{N}\right) \mu_{\partial}=0
$$

is satisfied. The vector field $V \in \Gamma(T M)$ is of Sobolev class $H^{1}$ on $M$ and if $\Phi \in C_{\nu}^{k-1}(M)$ and $\left.Z\right|_{\partial M}$ is of class $C_{\nu}^{k}$ (for $\left.k \geq 1\right), V$ can be chosen of class $C_{\nu}^{k}$ on $M$. 
For the special case $\left.Z\right|_{\partial M} \equiv 0$ this the problem is studied in [vWa]. As noted in that work its solvability is of great interest for investigations in fluid dynamics. The more general problem, prescribing the boundary value $\left.Z\right|_{\partial M}$ arbitrarily - modulo demanding consistency with the integrability condition (5.9) - has (to the author's knowledge) not been studied in the literature, but might also be of some importance for fluid dynamics. Another range of applications is electro-dynamics. One has to observe, however, that the the physical nature of this theory is such that harmonic solutions are preferable, except in very special gauges.

Similar to (5.7) also the action of other first order differential operators (the rotation and the gradient) can be expressed in terms of the exterior derivative and the co-differential. In principle it is possible to study coresponding boundary value problems with the same technique, we used here. One needs, however, to apply the Morrey-Kodeira decomposition (theorem 4) not only to zero-forms but to the space $\Omega^{r}(M)$, what yields some technical difficulties and will not be pursued here further.

\section{Acknowledgement :}

I gratefully acknowledge a discussions with W. von Wahl, on the special problem div $U=\Phi$ with $\left.U\right|_{\partial M}=0$, which inspired me to consider also the general question. 


\section{List of References}

[AMR] R.Abraham, J.E.Marsden and T.Ratiu, Manifolds, Tensor Analysis and Applications (2nd Edition, Springer-Verlag, New York, 1988).

[Bog] M.E.Bogovskij, Solutions of the first boundary value problem for the equa-

tions of continuity of an incompressible medium, Sov.Math.Dokl. 20 (1979) 1094.

[Con] P.E.Conner, The Neumann's problem for differential forms on Rientinannian manifolds, Mem.Am.Math.Soc. 20 (1956) 56.

[Duf] G.F.D.Duff, On the Potential Theory of Coclosed Harmonic Forms, Can.J.Math. 7 (1955) 127.

[DuSp] G.F.D.Duff and D.C.Spencer, Harmonic tensors on Riemannian manifolds with boundary, Ann.Math. 56 (1952) 128.

[GHV] W.Greub, S.Hâlperin and J.Vanstone, Connection, Curvature and Cohomology, Vol I (Academic Press, New York, 1972).

[Hir] M.W.Hirsch, Differential Topology, (Springer-Verlag, Berlin 1976).

[Hör] L.Hörmander, Linear Partial Differential Operators (Springer-Verlag, Berlin, 1969).

[Kre] R.Kress, Potentialtheoretische Randwertprobleme bei Tensorfeldern beliebiger Dimension und beliebigen Ranges, Arch.Rat.Mech.An. 47 (1972) 59.

[Mar] E.Martensen, Potentialtheorie (B.G. Teubner, Stuttgart, 1968).

[Mat] Y.Matsuchima, Vector bundle valued canonical forms, Osaka J.Math. 8 (1971) 309.

[Mo56] C.B.Morrey, A variational method in the theory of harmonic integrals II, Amer.J.Math. 78 (1956) 137.

[Mo62] C.B.Morrey, Mulitiple Integral in the Calculus of Variation (SpringerVerlag, New York, 1966).

[Pal] R.S.Palais, Seminar on the Atiyah-Singer Index Theorem, Chapter X (Princeton University Press 1965).

[Sch] G.Schwarz, The existence of a symmetric stress tensor in a non-local description of continuum mechanics, Mannheimer Math.Manuskr. 126/1991.

[vWa] W. von Wahl, On necessary and sufficient conditions for the solvability of the equations $\operatorname{rot} u=\gamma$ and $\operatorname{div} u=\epsilon$ with $u$ vanishing on the boundary, in : Lecture Notes in Mathematics 1431 (Ed.:J.G.Heywood e.a., SpringerVerlag, Berlin, 1990). 